\title{
An automation system based on labview to control the test of mechanical flow meters
}

\author{
J. Martínez ${ }^{1}$, R. Alvarez ${ }^{1}$, P. Cortez ${ }^{1}$, V. Mejia ${ }^{1}$ \& V. H. Zarate $^{2}$ \\ ${ }^{I}$ Mexican Institute of Water Technology (IMTA), Mexico \\ ${ }^{2}$ Technological Institute of Superior Studies of Monterrey \\ (ITESM), Mexico
}

\begin{abstract}
We present a computational system, based on a PC and LabVIEW to test and characterize the response of mechanical flow meters of conical cup type with vertical axis. A mechanical flow meter is a device used mainly to measure and calculate velocity of water flow on rivers and open channels.

These devices, suffer mechanical imperfections, over time, which is why it is important to calibrate them, normally twice a year, depending on its time of use. At the Mexican Institute of Water Technology (IMTA in Spanish) was designed and developed a circular water tank for the purpose of testing these meters.

This paper shows the automation systems designed to control the tests to calibrate these mechanical meters. The system is based on LabVIEW. LabVIEW is a general purpose programming tool with extensive libraries for data acquisition instrument control, data analysis, and data presentation. With this tool and a special hardware interface, it was possible to automate the process to test these mechanical meters.

The system is called SCM (system for characterization of mechanical meters). SCM controls the test of two mechanical meters simultaneously, and has some user control features that permit the Operator an easy to use human machine interface.

Keywords: mechanical flow meters, testing laboratory, LabVIEW based automation system.
\end{abstract}




\section{Introduction}

In 1996, the Mexican Institute of Water Technology built a water channel laboratory to test and calibrate the mechanical meters that are used all around Mexico [1]. These mechanical meters are very robust and priceless, that is the reason why they are very common in many countries. Figure 1 shows a typical mechanical flow meter.

The flow mechanical meters of conical cup type with vertical axis are devices that convert the velocity of flow water into revolutions by minute. This effect is produced because the force produced by the flow water into the conical cup converts velocity into counts of rotations. At each rotation a mechanical contact, inside of a contact chamber, close momentarily. This relationship between velocity and revolution per minute (RPM) is a characteristic obtained by experiments [2]. The purpose of the water tank laboratory is to obtain the characteristics of each mechanical meter. To test, two mechanical meters are placed at the end of the rotary metallic arm. This arm moves around the channel producing the same effect of water stream flow.

The circular water channel laboratory has $12 \mathrm{~m}$ of diameter, $80 \mathrm{~cm}$ of cross section and $1.40 \mathrm{~m}$ depth. The channel is associated to a mechanical arm moved by a motor of $15 \mathrm{HP}$. At the end of the arms, there exists two vertical axes to carry up one mechanical meter on each [1]. A picture of this channel is shown in figure 2. This circular channel is the first of its type, as show the different water channels, normally linear and 100 or $200 \mathrm{~m}$ long [3]. This circular channel represents a real challenge in this kind of laboratory.

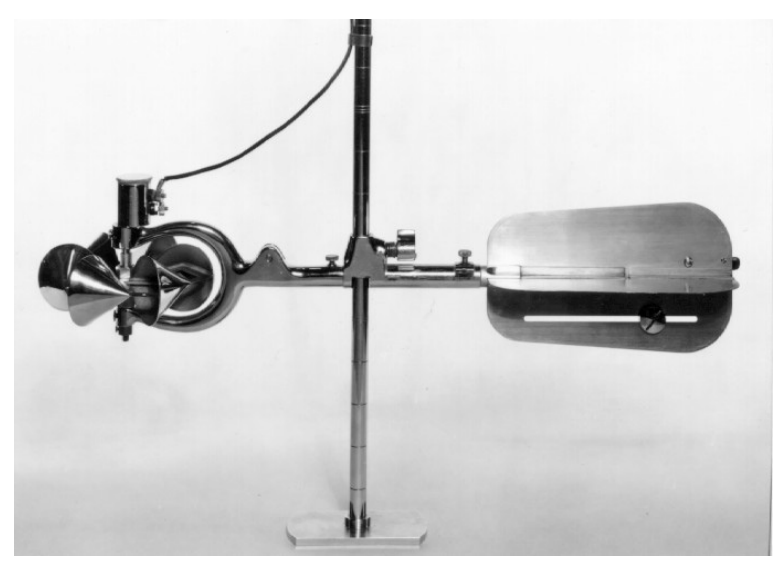

Figure 1: A typical mechanical meter of conical cup type. 


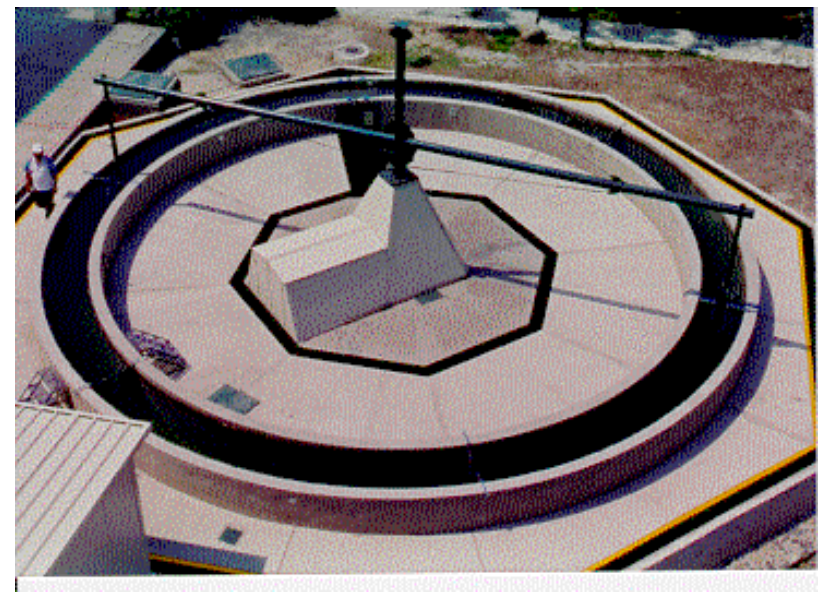

Figure 2: $\quad$ A picture of the circular channel.

\section{Methodology}

The objective of the system was to create an automation system easy to use, and flexible, in a way that all the different versions of mechanical meters could be characterized. At the same time, the resulting system must be capable of performing as good a calibration as would be made by a linear channel. We used a top-down methodology to arrive at design and development of the SCM system:

i) First of all, we analyze the different requirements,

ii) We decompose our problem in two subsystems: hardware and software

iii) We select the architecture for each subsystem

iv) We design and development each subsystem

v) We integrate and test the two subsystems

vi) We had a stage of testing by the end user: the Operator

The next sections show the design of the two main subsystems: software \& hardware.

\section{The system for characterization of mechanical meters (SCM)}

This systems mission is to control all the processes to characterize the mechanical meters. In figure 3 we show the architecture of the SCM. 


\subsection{The computational subsystem (software)}

The functions of the subsystem are:

i) To control the characterize process of two simultaneous mechanical meters with the help of a man-machine interface.

ii) To manage the data base with the history of the mechanical meters tested

iii) To control the different velocities of testing based on the control of a motor drive, from 0.14 to $3 \mathrm{~m} / \mathrm{s}$ with 12 different velocities.

iv) To calculate the characteristic equation velocity vs. RPM for each mechanical meter under test.

v) To generate the graphs, the reports and the tables for every mechanical meter tested.

vi) To eliminate the electrical spurious bounces.

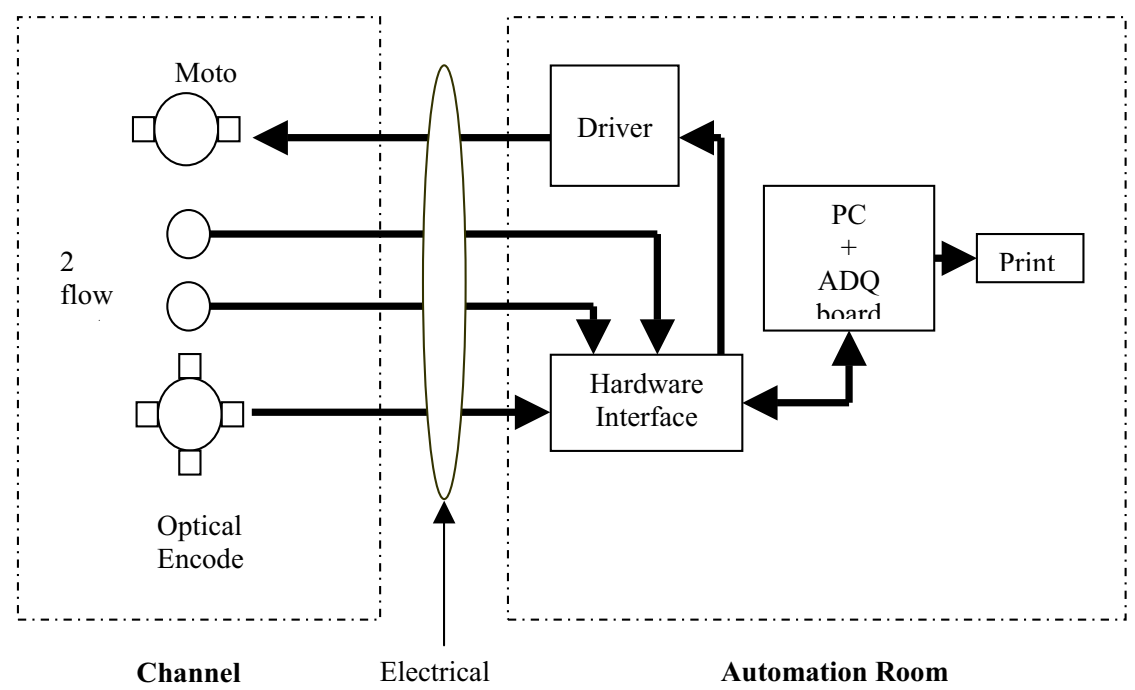

Figure 3: $\quad$ Architecture of the SCM.

\subsubsection{Control the characterizing process}

LabVIEW is a very easy to use language for creating man machine interfaces in automation systems. The system consists of different dialog menus to help the Human Operator control the test of two different mechanical meters. The principal program follows the next sequence:

Initialize all different parameters

\section{REPEAT}

Select option

IF option is characterize the meters:

Ask for the different parameters: time and velocity of test 


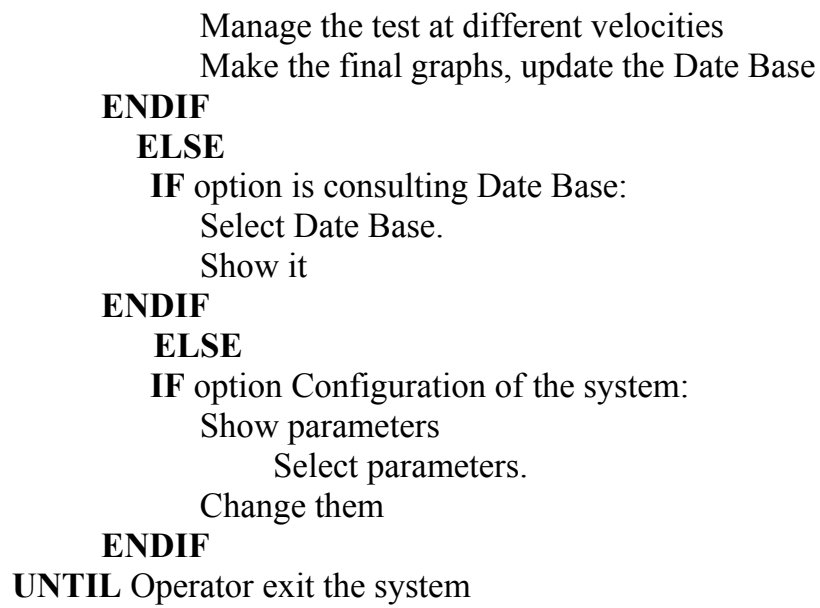

The full system based LabVIEW has approximately 64 different routines.

\subsubsection{Manage the data base}

The Date Base is an array of registers, one for each mechanical meter. The SCM can make updates to each register, based on the parameters read during the test. Also, it is possible for the Operator to review and update some of the fields on the register, for example:

i) Details of the maintenance made,

ii) Date of entrance to the laboratory

iii) Owner

iv) Special features

\subsubsection{Control the different velocities}

This characteristic permits the selection of 12 different velocities in a wide range, from 0.14 to $3 \mathrm{~m} / \mathrm{s}$. Also, performs global or individual tests. The global test means that the system automatically begin at the lower velocity and step by step reach the highest velocity. In each step, the system makes the arrangement to control the velocity of test, and also, it reads the train of pulses of the two mechanical meters.

It is possible also to perform individual tests. In this mode, the Operator selects one special velocity and the system executes all the process automatically, including the steps to move the arm to reach the velocity. It is important to say that the final velocity is reached following the dynamic conditions of the motor and its load.

\subsubsection{Calculate the characteristic equation velocity vs. RPM}

When the process of testing is finished, the arm's movement stops, then the system calculates, based on the data read, the equation of the line. 
Two parameters are calculated, the slop " $m$ " and the intersection " $b$ ". We use the function that LabVIEW has for this purpose, and the system graphs this result.

\subsubsection{Generate the graphs, the reports and the tables}

Finally, the SCM system generates a table, based on the equation of the line calculated. This table is practically the tabular representation of the graph. It is important because this table is the tool people will use when they use this mechanical meter in the field. In figure 4 we show one typical graph.

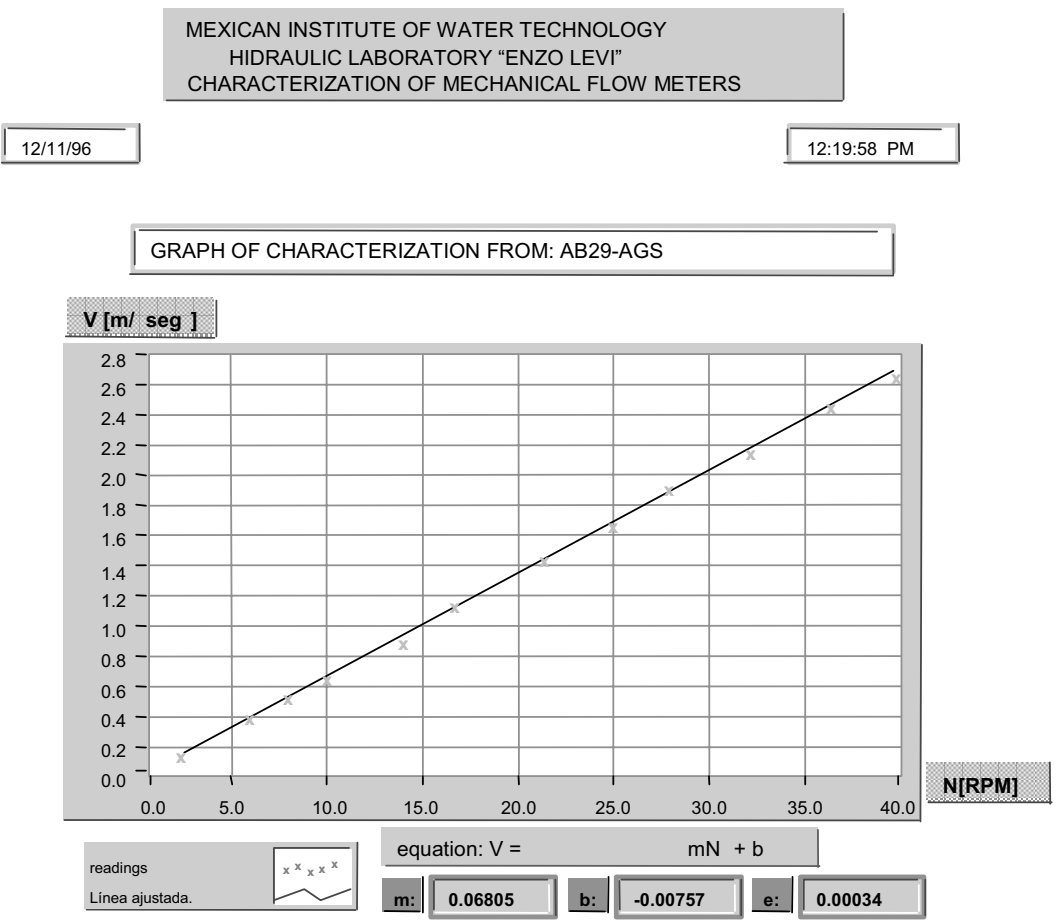

Figure 4: Typical graph based on the incoming data.

At the end of this process, the system prints out this information.

\subsubsection{Elimination of the electrical bounces}

The process to convert the revolutions per minute produced by the stream flow into the electrical train of pulses consists of injecting an electrical current through the mechanical movement of the meter to transform the revolution by minute into a train of electrical pulses. In this process, electrical bounces appear because of the mechanical nature of the contacts. A typical pulse is shown in figure 5. The problem arrives because the range of velocities that the mechanical meters measure is wide, from 0.14 to $3 \mathrm{~m} / \mathrm{s}$. With this range of velocity, the frequency of the electrical pulse vary from $0.2 \mathrm{~Hz}$ to $5 \mathrm{~Hz}$. 


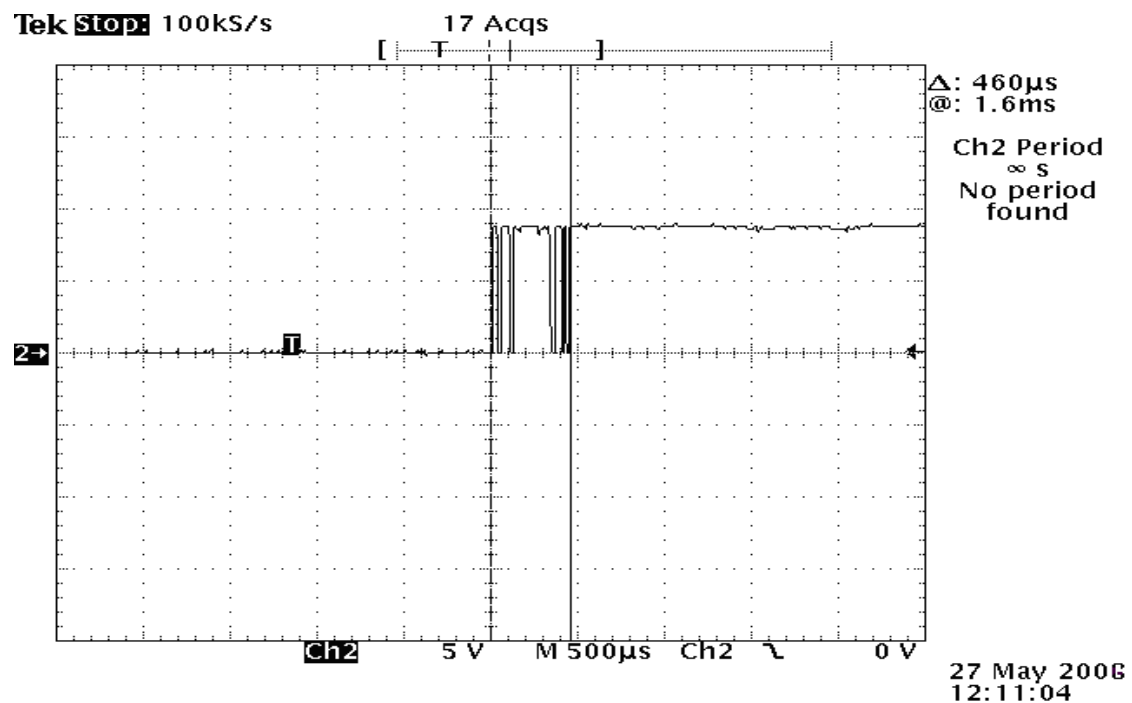

Figure 5: Noisy electrical signal produced by the mechanical flow meter when the contact close.

As seen in figure 5, the train of pulses is associated with a train of electrical pulses from the mechanical nature of the meter. These pulses have a random behavior, however based on experiments; we found that it has some characteristics that we can use to eliminate this electrical noise:

i) The maximal frequency of rotation is $300 \mathrm{RPM}$, at a period of $200 \mathrm{~ms}$.

ii) The minimal frequency is $12 \mathrm{RPM}$, at a period of $5 \mathrm{~s}$.

iii) The duty cycle between the time the contacts are closed and opened is less than $30 \%$.

iv) The maximum time the electrical pulses appear at the maximal frequency is less than $10 \mathrm{~ms}$.

v) The maximal time the electrical pulses appear at the minimal frequency is less than $300 \mathrm{~ms}$.

The analysis of this data shows that, for low frequency, the electrical spurious pulses are equivalent to a train of pulses generated by the mechanical meter at high frequency. If we decided to eliminate that noise with a low-pass filter, this filter could eliminate also valid pulses. This problem is amplified because every mechanical meter has its own response. To solve this problem, we need an adaptable filter, based on an estimated frequency of rotation, which is able to eliminate the spurious noise. We can then calculate the right rotary frequency of the mechanical meter. To implement this dynamic filter, we use two different approaches depending on the system, as we will see in the next section. 
To build the dynamic filter, we use the knowledge related to the behavior of this quasi-random noise. Furthermore, we know the velocity of testing because we control the rotary electrical motor. Our algorithm takes into account these two factors and adapts the parameter of the filter to eliminate these electrical spurious pulses. The algorithm is, for a specific velocity of test, shown in figure 6 , on the next page.

Control the electrical motor to put the testing velocity

Put the filter parameters based on the velocity

\section{REPEAT}

Monitoring the pulse arrival encoder and modify if necessary

Monitoring the actual velocity with the optical

IF pulse arrives:

the filter parameter.

Check if the period of arrives was larger than

IF yes, pulse is ok, count it

ENDIF

ELSE ignore pulse

UNTIL time of test ends or Operator stops the test

Figure 6: Algorithm to denounce electrical noisy signals.

\subsection{The electronic subsystem (hardware)}

The hardware consists of a PC Pentium II $400 \mathrm{MHz}$ computer associated to a dispositive date acquisition (DAQ) card. We use the PCI-6071-E of National Instruments, and it is charged to communicate with our homemade interface board. Finally, we have a drive to control the motor. We use the ACS 501, adjustable frequency (ABB mark) driver

We designed an electronic board to interface the different devices to the dispositive date acquisition (DAQ) card. The elements and function of the interface are:

i) Injection of electrical current at each mechanical meter in test.

ii) Reading of two asynchronous train of electrical pulses, each one may have different behavior

iii) Generation of different electrical control signals to the motor drive

iv) Reading the different electrical status signals from the motor drive

v) Reading the train of pulses generated by the optical encoder to measure the actual velocity of movement

\section{Results and conclusions}

This way of making the characterizations by means of the annular channel is not very common since by regulating the characterizations were made in straight 
channels. The construction of the system and its automatization are an innovation of the technology to characterize flow meters in short times and with greater precision and trustworthiness. In order to verify the effectiveness of the developed system I am made a comparative experimental study of the characterization of a lot of flow meters, as much in the annular channel as in the certificate channel of the laboratory of hydraulics of Canada Centers for Inland Waters (CCIW) the obtained characteristic equations in both channels for each flow meters are very similar, which is translated in acceptable uncertainties of the annular channel like characterization system with which we can say that the results that are obtained are correct. The characteristics of the system are:

i) Easy of use

ii) One hour per test,

iii) Easy of manage the Data Base y Make statistical analysis

Right now we are preparing the laboratory of test to achieve an International Certification.

\section{References}

[1] Petronílo Cortés, Ricardo Alvarez. "Circular water channel for testing mechanical flow meters" In Spanish. XIV Congreso Nacional de Hidráulica. Asociación Mexicana de Hidráulica. Acapulco Gro..pp. 1-5, October 1996.

[2] U.S. Department of the Interior. "Water Measurement Manual "third edition 1997 (these web pages are based on the third edition) http://www.usbr.gov/wrrl/fmt/wmm/ [consulted November 1999].

[3] The Canada Centre for Inland Waters. World Wide Web servers at Burlington, Ontario. http://www.cciw.ca/ [consulted November 1999]. 